\title{
Numerical analysis of Richards' problem for water penetration in unsaturated soils
}

\author{
A. Barari ${ }^{1}$, M. Omidvar ${ }^{2}$, A. R. Ghotbi ${ }^{3}$, and D. D. Ganji \\ ${ }^{1}$ Departments of Civil and Mechanical Engineering, Babol University of Technology, \\ Babol, Iran \\ ${ }^{2}$ Faculty of Engineering, University of Golestan, Gorgan, Iran \\ ${ }^{3}$ Department of Civil Engineering, Shahid Bahonar University, Kerman, Iran
}

Received: 5 September 2009 - Accepted: 14 September 2009 - Published: 12 October 2009

Correspondence to: A. Barari (amin78404@yahoo.com)

Published by Copernicus Publications on behalf of the European Geosciences Union.

6359

\begin{abstract}
Unsaturated flow of soils in unsaturated soils is an important problem in geotechnical and geo-environmental engineering. Richards' equation is often used to model this phenomenon in porous media. Obtaining appropriate solution to this equation there5 fore provides better means to studying the infiltration into unsaturated soils. Available methods for the solution of Richards' equation mostly fall in the category of numerical methods, often having restrictions for practical cases. In this research, two analytical methods known as Homotopy Perturbation Method (HPM) and Variational Iteration Method (VIM) have been successfully utilized for solving Richards' equation. Results 10 obtained from the two methods mentioned show a remarkably high precision in the obtained solution, compared with the existing exact solutions available.
\end{abstract}

\section{Introduction}

Modeling water flow through porous media presents an important problem of practical interest for geotechnical and geo-environmental engineering, as well as many other 15 areas of science and engineering. Study of this phenomenon requires proper formulation of the governing equations and constitutive relations involved. Currently, equations used for describing fluid flow through porous media are based mainly on semi-empirical equations first derived by Buckingham (1907) and Richards (1931). Despite limitations and drawbacks, Richards' equation is still the most widely used equation for model-

20 ing unsaturated flow of water through soil (porous media) (Hoffmann, 2003). Due to the importance and wide applications of the problem, many researches have been devoted in the past to proper assessment of different forms of Richards' equation. Both analytical and numerical solutions have been investigated in the literature. Analytical solutions to Richards' equation are rather scarce and are generally limited to only special cases (Ju and Kung, 1997; Arampatzis et al., 2001). This is mainly due to the dependence of hydraulic conductivity and diffusivity - two important parameters in the 
equation - on moisture content, combined with the non-trivial forcing conditions that are often encountered in engineering practice (Ju and Kung, 1997; Arampatzis et al., 2001; Kavetski et al., 2002). As a result, application of many numerical methods to the solution of Richards' equation with various engineering applications has been in-

5 vestigated in the literature. Finite element and finite difference methods have been adopted by several researchers (Clement et al., 1994; Baca et al., 1997; Bergamaschi and Putti, 1999; Milly, 1985). Mass lumping was employed in these studies to improve stability. Time stepping schemes such as the Douglas-Jones-predictor-corrector method, Runge-Kutta method and backward difference formulae should also be men-

10 tioned in this context (Kavetski et al., 2001a; Miller et al., 2005). Tabuada et al. (1995) used an implicit method and presented equations governing two-dimensional irrigation of water into unsaturated soil based on Richards' equation. The Gauss-Seidel method was then effectively used to solve the resulting equations. Ross (2003) introduced an efficient non-iterative solution for Richards' equation using soil property descriptions as

15 proposed by Brooks and Corey (1964). In his method, Ross used a space and time discretization scheme in order to derive a tridiagonal set of linear equations which were then solved non-iteratively. Varado et al. (2006) later conducted a thorough assessment of the method proposed by Ross and concluded that the model provides robust and accurate solutions as compared with available analytical solutions (Basha, 1999).

20 Several other iterative solutions have also been cited in the literature. One such study is that of Farthing et al. (2003) which used the well-known pseudo-transient continuation approach to solve the nonlinear transient water infiltration problem, as well as the steady-state response as governed Richards' equation. Other commonly used iterative schemes include the Picard iteration scheme (Chounet et al., 1999; Forsyth et al.,

25 1995), the Newton and inexact Newton schemes (Jones and Woodward, 2001; Kavetski et al., 2001b; Kees and Miller, 2002) and hybrid Newton-Picard methods. Huang et al. (1996) considered the modified Picard iteration schemes and presented several convergence criteria as to evaluate the efficiency of the various iterative methods. In geo-environmental applications, Bunsri et al. (2008) solved Richards' equation accom-

6361

panied by advective-dispersive solute transport equations by the Galerkin technique. Witelski (1997) used perturbation methods to study the interaction of wetting fronts with impervious boundaries in layered soils governed by Richards' equation. Through comparison with numerical solutions, Witelski concluded that perturbation methods are 5 able to yield highly accurate solutions to Richards' equation (Witelski, 1997).

Each method mentioned above encounters Richards' equation in a certain way. Often, assumptions are made and empirical models are implemented in order to overcome difficulties in solving the equation due to high interdependence of some of the parameters involved. Analytical solutions often fit under classical perturbation meth10 ods (Kevorkian and Cole, 1996; Nayfeh, 1973; Nayfeh and Mook, 1979). However, as with other analytical techniques, certain limitations restrict the wide application of perturbation methods, most important of which is the dependence of these methods on the existence of a small parameter in the equation. Disappointingly, the majority of nonlinear problems have no small parameter at all. Even in cases where a small

15 parameter does exist, the determination of such a parameter doesn't seem to follow any strict rule, and is rather problem-specific. Furthermore, the approximate solutions solved by the perturbation methods are valid, in most cases, only for the small values of the parameters. It is obvious that all these limitations come from the small parameter assumption. In the present study, two powerful analytical methods Homotopy Perturba20 tion Method (HPM) (He, 2003, 1999a, 2006a, 2000; Barari et al., 2008a,b; Ghotbi et al., 2008a,b) and Variational Iteration Method (VIM) (He, 1997, 1999b, 2006b; Sweilam and Khader, 2007; Momani and Abuasad, 2006; Barari et al., 2008c) have been employed to solve the problem of one-dimensional infiltration of water in unsaturated soil governed by Richards' equation. HPM is actually a coupling of the perturbation method 25 and Homotopy Method, which has eliminated limitations of the traditional perturbation methods. HPM requires no small parameters in the equations and can readily eliminate the limitations of the traditional perturbation techniques. He $(\mathrm{He}, 1997,1999 \mathrm{~b}$, $2006 \mathrm{~b})$ proposed a variational iteration method (VIM) based on the use of restricted variations and correction functionals which has found a wide application for the so- 
lution of nonlinear ordinary and partial differential equations. This method does not require the presence of small parameters in the differential equation, and provides the solution (or an approximation to it) as a sequence of iterates. The method does not require that the nonlinearities be differentiable with respect to the dependent variable

5 and its derivatives.

In the next sections, Richards' equation and the relative models involved are introduced, followed by a thorough explanation of the analytical methods used to solve the equation. Illustrative examples are also given in order to demonstrate the effectiveness of the method in solving Richards' equation. The examples considered, despite involv-

10 ing relatively simple one-dimensional cases, represent a nonlinear problem. Despite the major advances of science in the field of solving differential equations, it is still very difficult to solve such nonlinear problems either numerically or analytically. He and Lee (2009) attributes this shortcoming to the fact that various discredited methods and numerical simulations apply iteration techniques to find numerical solutions of nonlinear

15 problems, and since nearly all iterative methods are sensitive to initial solutions, it is very difficult to obtain converged results in cases of strong nonlinearity.

\section{Richards' equation}

The basic theories describing fluid flow through porous media were first introduced by Buckingham (1907) who realized that water flow in unsaturated soil is highly dependent 20 on water content. Buckingham introduced the concept of "conductivity", dependent on water content, which is today known as unsaturated hydraulic conductivity (after Rolston, 2007). This equation is usually referred to as Buckingham law (Narasimhan, 2005). Buckingham also went on to define moisture diffusivity which is the product of the unsaturated hydraulic conductivity and the slope of the soil-water characteristic curve. Nearly two decades later, Richards (1931) applied the continuity equation to Buckingham's law - which itself is an extension of Darcy's law - and obtained a general partial differential equation describing water flow in unsaturated, non-swelling soils

6363

with the matric potential as the single dependent variable (Philip, 1974). There are generally three main forms of Richards' equation present in the literature namely the mixed formulation, the $h$-based formulation and the $\theta$-based formulation, where $h$ is the weight-based pressure potential and $\theta$ is the volumetric water content.

5 Since Richards' equation is a general combination of Darcy's law and the continuity equation as previously mentioned, the two relations must first be written in order to derive Richards' equation. Herein, one-dimensional infiltration of water in vertical direction of unsaturated soil is considered, for which Darcy's law and the continuity equation are given by Eqs. (1) and (2) respectively:

$10 \quad q=-K \frac{\partial H}{\partial z}=-K \frac{\partial(h+z)}{\partial z}=-K\left(\frac{\partial h}{\partial z}+1\right)$

and

$\frac{\partial \theta}{\partial t}=-\frac{\partial q}{\partial z}$

where $K$ is hydraulic conductivity, $H$ is head equivalent of hydraulic potential, $q$ is flux density and $t$ is time. The mixed form of Richards' equation is obtained by substituting 15 Eq. (1) in Eq. (2):

$\frac{\partial \theta}{\partial t}=\frac{\partial}{\partial z}\left[K\left(\frac{\partial h}{\partial z}+1\right)\right]$.

Equation (3) has two independent variables: the soil water content, $\theta$, and pore water pressure head, $h$. Obtaining solutions to this equation therefore requires constitutive relations to describe the interdependence among pressure, saturation and hydraulic 20 conductivity. However, it is possible to eliminate either $\theta$ or $h$ by adopting the concept of differential water capacity, defined as the derivative of the soil water retention curve:

$C(h)=\frac{\mathrm{d} \theta}{\mathrm{d} h}$ 
The $h$-based formulation of Richards' equation is thus obtained by replacing Eq. (4) in Eq. (3):

$C(h) \times \frac{\partial h}{\partial t}=\frac{\partial}{\partial z}\left(K \frac{\partial h}{\partial z}\right)+\frac{\partial K}{\partial z}$.

This is a fundamental equation in geotechnical and geo-environmental engineering 5 and is used for modeling flow of water through unsaturated soils. For instance, the twodimensional form of the equation can be used to model seepage in the unsaturated zone above water table in an earth dam.

Introducing a new term $D$, pore water diffusivity, defined as the ratio of the hydraulic conductivity to the differential water capacity, the $\theta$-based form of Richards' equation may be obtained. $D$ can therefor be written as:

$D=\frac{K}{C}=\frac{K}{\frac{\mathrm{d} \theta}{\mathrm{d} h}}=K \frac{\mathrm{d} h}{\mathrm{~d} \theta}$.

It should be noted that both $D$ and $K$ are highly dependent on water content. Combining Eq. (6) with Eq. (3) gives Richards' equation as:

$\frac{\partial \theta}{\partial t}=\frac{\partial}{\partial z}\left(D \frac{\partial \theta}{\partial z}\right)+\frac{\partial K}{\partial z}$.

15 In order to solve Eq. (7), one must first properly address the task of estimating $D$ and $K$, both of which are dependent on water content. Several models have been suggested for determining these parameters. The Van Genuchten model (Van Genuchten, 1980) and Brooks and Corey's model (Brooks and Corey, 1964; Corey, 1994) are the more commonly used models. The Van Genuchten model uses mathematical rela20 tions to relate soil water pressure head with water content and unsaturated hydraulic conductivity, through a concept called "relative saturation rate". This model matches experimental data but its functional form is rather complicated and it is therefore difficult to implement it in most analytical solution schemes. Brooks and Corey's model on

6365

the other hand has a more precise definition and is therefore adopted in the present research. This model uses the following relations to define hydraulic conductivity and water diffusivity:

$$
\begin{aligned}
D(\theta) & =\frac{K_{\mathrm{s}}}{\alpha \lambda\left(\theta_{\mathrm{s}}-\theta_{\mathrm{r}}\right)}\left(\frac{\theta-\theta_{\mathrm{r}}}{\theta_{\mathrm{s}}-\theta_{\mathrm{r}}}\right)^{2+\frac{1}{\lambda}} \\
5 \quad K(\theta) & =K_{\mathrm{s}}\left(\frac{\theta-\theta_{\mathrm{r}}}{\theta_{\mathrm{s}}-\theta_{\mathrm{r}}}\right)^{3+\frac{2}{\lambda}} .
\end{aligned}
$$

Where $D(\theta)$ and $K(\theta)$ represent diffusivity and conductivity, respectively, $K_{\mathrm{s}}$ is saturated conductivity, $\theta_{\mathrm{r}}$ is residual water content, $\theta_{\mathrm{s}}$ is saturated water content and $\alpha$ and $\lambda$ are experimentally determined parameters. Brooks and Corey determined $\lambda$ as pore-size distribution index (Brooks and Corey, 1964). A soil with uniform pore-size 10 possesses a large $\lambda$ while a soil with varying pore-size has small $\lambda$ value. Theoretically, the former can reach infinity and the latter can tend towards zero. Further manipulation of Brooks and Corey's model yields the following equations (Witelski, 1997; Corey, 1986; Witelski, 2005):

$$
\begin{aligned}
D(\theta) & =D_{0}(n+1) \theta^{n} \quad n \geq 0 \\
15(\theta) & =K_{0} \theta^{k} \quad k \geq 1
\end{aligned}
$$

where $K_{0}, D_{0}$ and $k$ are constants representing soil properties such as pore-size distribution, particle size, etc. In this representation of $D$ and $K, \theta$ is scaled between 0 and 1 and diffusivity is normalized so that for all values of $m, \int D(\theta) \mathrm{d} \theta=1$ (after Nasseri et al., 2008). Equation (11) suggests that conductivity may have linear, parabolic, cubic, etc. variation with water content, associated with $k$ values of $1,2,3$, etc., respectively.

Several analytical and numerical solutions to Richards' equation exist based on Brooks and Corey's representation of $D$ and $K$. Replacing $n=0$ and $k=2$ in Eqs. (10) and (11) yields the classic Burgers' equation extensively studied by many researchers (Basha, 2002; Broadbridge and Rogers, 1990; Whitman, 1974). The gen- 
eralized Burgers' equation is also obtained for general values of $k$ and $n$ (Whitman, 1974).

As seen previously, the two independent variables in Eq. (7) are time and depth. By applying the traveling wave technique (Wazwaz, 2005; He, 1997; Elwakil et al., 5 2004), instead of time and depth, a new variable which is a linear combination of them is found. Tangent-hyperbolic function is commonly applied to solve these transform equations (Soliman, 2006; Abdou and Soliman, 2006). Therefore the general form of Burgers' equation in order of $(n, 1)$ is obtained as (Wazwaz, 2005):

$$
\frac{\partial \theta}{\partial t}+\alpha \theta^{n} \frac{\partial \theta}{\partial z}-\frac{\partial^{2} \theta}{\partial z^{2}}=0
$$

10 The exact solution to Eq. (12) can be found to be:

$$
\begin{aligned}
\theta(z, t) & =\left(\frac{\gamma}{2}+\frac{\gamma}{2} \tanh \left(\left[A_{1}\left(z-A_{2} t\right)\right]\right)^{1 / n}\right) \\
A_{1} & =\frac{-\alpha n+n|\alpha|}{4(1+n)} \gamma \quad(n \neq 0) \\
A_{2} & =\frac{\gamma \alpha}{1+n}
\end{aligned}
$$

$\gamma$ is an arbitrary coefficient which is selected as 1 here, following Nasseri et al. (2008).

15 In this study, nonlinear infiltration of water in unsaturated soil has been studied using Richards' equation and by employing Brooks and Corey's model to represent hydraulic conductivity and diffusivity. The problems discussed herein may be generalized to any combination of $(k, n)$, having different physical interpretations. However, as Witelski (1997) mentioned, the case of $k=n+1$ provides certain analytical simplifications, and will therefore be considered here. The conductivity function has been selected in two independent example cases as $\theta^{2} / 2$ and $\theta^{3} / 3$ which corresponds to parabolic and cubic variation of conductivity with $k=2$ and $k=3$, respectively. The linear variation of conductivity, i.e., $k=1$ represents a linear problem and has already been thoroughly studied by Witelski (1997). The respective $n$ values of one and two are associated 6367

with the above chosen conductivities. The values considered represent fine-textured to medium-textured soils. The resulting form of Richards' equation will therefore be:

$$
\frac{\partial \theta}{\partial t}=K_{0} \frac{\partial}{\partial z}\left(\theta^{n+1}\right)+D_{0} \frac{\partial^{2}}{\partial z^{2}}\left(\theta^{n+1}\right)
$$

Witelski (1997) reasoned that nonlinear variations of conductivity means that steady5 profile traveling wave solutions will exist in the solutions that move with constant speed and balance conductive transport and dispersal. The profiles do not vary with time, unlike the linear problem which has a diffusive nature.

Homotopy perturbation method (HPM) (He, 2006a, 2000; Barari et al., 2008a,b; Ghotbi et al., 2008a,b) and variational iteration method (VIM) (He, 1997, 1999b, 2006b;

10 Sweilam and Khader, 2007; Momani and Abuasad, 2006; Barari et al., 2008c) described below have been used to solve Eq. (12). HPM and VIM are first introduced briefly, and are then implemented in order to solve Richards' equation as described by Eq. (7) and supported by Eqs. (10) and (11).

\section{Basic idea of He's homotopy perturbation method (HPM)}

15 Simply put, solving a nonlinear problem by means of HPM begins with a trial-function of the same order of the original problem with some unknown parameters, followed by constructing a linear differential equation whose solution is the chosen trial-function. The next step is to construct such a homotopy that when the homotopy parameter $p=0$, it becomes the above constructed linear equation; and when $p=1$, it turns out to

20 be the original nonlinear equation. The changing process of $p$ from zero to unity is just that of the trial-function (initial solution) to the exact solution. To approximately solve the problem, the solution is expanded into a series of $p$, just like that of the classical perturbation method. Generally, one iteration is enough, but the solution is always obtained with three steps at most. 
To illustrate the basic ideas of HPM, we consider the following nonlinear differential equation:

$A(u)-f(r)=0, \quad r \in \Omega$,

with the boundary conditions of

$5 \quad B\left(u, \frac{\partial u}{\partial n}\right)=0, \quad r \in \Gamma$,

where $A, B, f(r)$ and $\Gamma$ are a general differential operator, a boundary operator, a known analytical function and the boundary of the domain $\Omega$, respectively.

Generally speaking the operator $A$ can be divided into a linear part $L$ and a nonlinear part $N(u)$. Equation (15) can therefore, be rewritten as:

$10 L(u)+N(u)-f(r)=0$,

By the Homotopy technique, we construct a homotopy $v(r, p): \Omega \times[0,1] \rightarrow R$, which satisfies:

$$
\begin{aligned}
H(v, p) & =(1-p)\left[L(v)-L\left(u_{0}\right)\right]+p[A(v)-f(r)]=0, \\
p & \in[0,1], \quad r \in \Omega,
\end{aligned}
$$

or

$H(v, p)=L(v)-L\left(u_{0}\right)+p L\left(u_{0}\right)+p[N(v)-f(r)]=0$.

where $p \in[0,1]$ is an embedding parameter, while $u_{0}$ is an initial approximation of Eq. (15), which satisfies the boundary conditions. Obviously, from Eqs. (18) and (19) we will have:

$20 \quad H(v, 0)=L(v)-L\left(u_{0}\right)=0$,

$H(v, 1)=A(v)-f(r)=0$.

6369

The changing process of $p$ from zero to unity is just that of $v(r, p)$ from $u_{0}(r)$ to $u(r)$. In topology, this is called deformation, while $L(v)-L\left(u_{0}\right)$ and $A(v)-f(r)$ are called homotopy.

According to the HPM, we can first use the embedding parameter $p$ as a "small 5 parameter", and assume that the solution of Eqs. (18) and (19) can be written as a power series in $p$ :

$v=v_{0}+p v_{1}+p^{2} v_{2}+\cdots$

Setting $p=1$ yields in the approximate solution of Eq. (18) to:

$u=\lim _{p \rightarrow 1} v=v_{0}+v_{1}+v_{2}+\cdots$

10 The combination of the perturbation method and the homotopy method is called the HPM, which eliminates the drawbacks of the traditional perturbation methods while keeping all its advantage.

The series (23) is convergent for most cases. However, the convergent rate depends on the nonlinear operator $A(v)$. Moreover, $\mathrm{He}(1999 \mathrm{a})$ made the following suggestions:

15 1. The second derivative of $N(v)$ with respect to $v$ must be small because the parameter may be relatively large, i.e. $p \rightarrow 1$.

2. The norm of $L^{-1} \frac{\partial N}{\partial v}$ must be smaller than one so that the series converges.

\section{Basic idea of variational iteration method (VIM)}

To clarify the basic ideas of VIM, we consider the following differential equation:

$20 L u+N u=g(t)$

where $L$ is a linear operator, $N$ is a nonlinear operator and $g(t)$ is an inhomogeneous term. 
According to VIM, we can write down a correction functional as follows:

$u_{n+1}(t)=u_{n}(t)+\int_{0}^{t} \lambda\left(L u_{n}(\tau)+N \tilde{u}_{n}(\tau)-g(\tau)\right) \mathrm{d} \tau$

Where $\lambda$ is a general lagrangian multiplier which can be identified optimally via the variational theory. The subscript $n$ indicates the $n$-th approximation and $u_{n}$ is considered 5 as a restricted variation, i.e. $\delta \tilde{u}_{n}=0$.

\section{Implementation of HPM to solve Richards' equation}

\subsection{Case 1: if $n=1$}

To solve Richard equation when $n=1$ by means of HPM, we consider the following process after separating the linear and nonlinear parts of the equation.

A homotopy can be constructed as follows:

$$
\begin{aligned}
H(v, p)= & (1-p)\left(\frac{\partial}{\partial t} v(z, t)-\frac{\partial}{\partial t} u_{0}(z, t)\right)+ \\
& p\left(\frac{\partial}{\partial t} v(z, t)+v(z, t) \frac{\partial}{\partial z} v(z, t)-\frac{\partial^{2}}{\partial z^{2}} v(z, t)\right) .
\end{aligned}
$$

Substituting $v=v_{0}+p v_{1}+\ldots$ in to Eq. (26) and rearranging the resultant equation based on powers of $p$-terms, one has:

${ }_{15} \quad p^{0}: \frac{\partial}{\partial t} v_{0}(z, t)=0$,

$p^{1}: \frac{\partial}{\partial t} v_{1}(z, t)+v_{0}(z, t) \frac{\partial}{\partial z} v_{0}(z, t)-\frac{\partial^{2}}{\partial z^{2}} v_{0}(z, t)=0$,

$p^{2}: \frac{\partial}{\partial t} v_{2}(z, t)-\frac{\partial^{2}}{\partial x^{2}} v_{1}(z, t)+v_{1}(z, t) \frac{\partial}{\partial z} v_{0}(z, t)+v_{0}(z, t) \frac{\partial}{\partial z} v_{1}(z, t)=0$,

With the following conditions:

$v_{0}(z, 0)=0.5-0.5 \tanh (0.25 z)$

$v_{i}(z, 0)=0 \quad i=1,2, \ldots$

With the effective initial approximation for $v_{0}$ from the conditions (30) and solutions of

5 Eqs. (27-29) may be written as follows:

$v_{0}(z, t)=0.5-0.5 \tanh (0.25 z)$,

$v_{1}(z, t)=\frac{1}{16} t-\frac{1}{16} t \tanh (0.25 z)^{2}$,

$v_{2}(z, t)=-\frac{1}{128} t^{2} \tanh (0.25 z)\left(-1+\tanh (0.25 z)^{2}\right)$,

In the same manner, the rest of components were obtained using the Maple package. According to the HPM, we can conclude that:

$\theta(z, t)=\lim v(z, t)=v_{0}(z, t)+v_{1}(z, t)+\ldots$,

$$
p \rightarrow 1
$$

Therefore, substituting the values of $v_{0}(z, t), v_{1}(z, t), v_{2}(z, t)$ from Eqs. (31-33) in to Eq. (34) yields:

${ }_{15} \theta(z, t)=0.5-0.5 \tanh (0.25 z)+\frac{1}{16} t-\frac{1}{16} t \tanh (0.25 z)^{2}-\frac{1}{128} t^{2} \tanh (0.25 z)$

$$
\left(-1+\tanh (0.25 z)^{2}\right)
$$

\subsection{Case 2: if $n=2$}

To solve Richard equation if $n=2$ by means of HPM, we consider the following process after separating the linear and nonlinear parts of the equation. 
A homotopy can be constructed as follows:

$$
\begin{aligned}
H(v, p)= & (1-p)\left(\frac{\partial}{\partial t} v(z, t)-\frac{\partial}{\partial t} u_{0}(z, t)\right)+ \\
& p\left(\frac{\partial}{\partial t} v(z, t)+v(z, t)^{2} \frac{\partial}{\partial z} v(z, t)-\frac{\partial^{2}}{\partial z^{2}} v(z, t)\right),
\end{aligned}
$$

Substituting $v=v_{0}+p v_{1}+\ldots$ in to Eq. (36) and rearranging the resultant equation based 5 on powers of $p$-terms, one has:

$p^{0}: \frac{\partial}{\partial t} v_{0}(z, t)=0$,

$p^{1}: \frac{\partial}{\partial t} v_{1}(z, t)+v_{0}(z, t)^{2} \frac{\partial}{\partial z} v_{0}(z, t)-\frac{\partial^{2}}{\partial z^{2}} v_{0}(z, t)=0$

$p^{2}:-\frac{\partial^{2}}{\partial z^{2}} v_{1}(z, t)+\frac{\partial}{\partial t} v_{2}(z, t)+2 v_{0}(z, t) v_{1}(z, t) \frac{\partial}{\partial z} v_{0}(z, t)$

$$
+v_{0}(z, t)^{2} \frac{\partial}{\partial z} v_{1}(z, t)=0
$$

10 With the following conditions:

$v_{0}(z, 0)=(0.5-0.5 \tanh (0.3333333 z))^{0.5}$

$v_{i}(z, 0)=0 \quad i=1,2, \ldots$

With the effective initial approximation for $v_{0}$ from the conditions (40) and solutions of Eqs. (37-39) may be written as follows:

$15 v_{0}(z, t)=(0.5-0.5 \tanh (0.3333333 z))^{0.5}$,

$v_{1}(z, t)=\frac{9 t\left(\begin{array}{l}463 \tanh (0.33333 z)^{3}- \\ 308641821 \tanh (0.33333 z)^{2}- \\ 463 \tanh (0.33333 z)+308641821\end{array}\right)}{50000000000 \sqrt{2-2(0.33333 z)}}$,

$$
\begin{aligned}
v_{2}(z, t)= & \frac{1}{0.4 \times 10^{24} \sqrt{2-2 \tanh (0.33333 z)}} \times \\
& \left(\begin{array}{l}
6.4819 \times 10^{15} \tanh (0.33333 z)^{5}+ \\
8.3336 \times 10^{15} \tanh (0.33333 z)^{4}- \\
1.8518 \times 10^{21} \tanh (0.33333 z)^{3}+ \\
6.1727 \times 10^{20} \tanh (0.33333 z)^{2}+ \\
1.8518 \times 10^{21} \tanh (0.33333 z)^{1}- \\
6.1728^{20}
\end{array}\right)
\end{aligned}
$$

In the same manner, the rest of components were obtained using the Maple package. According to the HPM, we can conclude that:

$5 \quad \theta(z, t)=\lim v(z, t)=v_{0}(z, t)+v_{1}(z, t)+\ldots$,

$$
p \rightarrow 1 \text {. }
$$

Therefore, substituting the values of $v_{0}(z, t), v_{1}(z, t), v_{2}(z, t)$ from Eqs. $(41-43)$ in to Eq. (44) yields:

$$
\theta(z, t)=(0.5-0.5 \tanh (0.3333333 z))^{0.5}+\frac{9 t\left(\begin{array}{l}
463 \tanh (0.33333 z)^{3}- \\
308641821 \tanh (0.33333 z)^{2}- \\
463 \tanh (0.33333 z)+308641821
\end{array}\right)}{50000000000 \sqrt{2-2(0.33333 z)}}
$$

$$
+\frac{1}{0.4 \times 10^{24} \sqrt{2-2 \tanh (0.33333 z)}} \times
$$




$$
\left(t^{2}\left(\begin{array}{l}
6.4819 \times 10^{15} \tanh (0.33333 z)^{5}+ \\
8.3336 \times 10^{15} \tanh (0.33333 z)^{4}- \\
1.8518 \times 10^{21} \tanh (0.33333 z)^{3}+ \\
6.1727 \times 10^{20} \tanh (0.33333 z)^{2}+ \\
1.8518 \times 10^{21} \tanh (0.33333 z)^{1}- \\
6.1728^{20}
\end{array}\right)\right)
$$

The obtained solution has been drawn in Figs. 1 and 2 for $n=1$ and $n=2$, respectively, along with the results of VIM from the following section, as well as the exact solution available. It is noteworthy from the figures that the plotted values of $\theta(z, t)$

5 include negative z-values as well as positive values, whereas Richards' equation usually has significant physical meaning for positive z-values. However, since there are some solutions given for infinite conditions in Richard's equation in the literature, i.e., for $-\infty<z<+\infty$ (especially for linear variations of $k$ ), solutions for negative values of $z$ have also been presented in the figures.

\section{Implementation of VIM to solve Richards' equation}

\subsection{Case 1: $n=1$}

To solve the Eq. (12) by means of VIM, one can construct the following correction functional:

$\theta_{n+1}(z, t)=\theta_{n}(z, t)+\int_{0}^{t} \lambda^{*}\left(\frac{\partial}{\partial \tau} \theta_{n}(z, \tau)+\theta_{n}(z, \tau) \frac{\partial}{\partial z} \theta_{n}(z, \tau)-\frac{\partial^{2}}{\partial z^{2}} \theta_{n}(z, \tau)\right) \mathrm{d} \tau$

15 Its stationary conditions can be obtained as follows:

$$
\begin{aligned}
\left.\lambda^{\prime *}\right|_{\tau=t} & =0, \\
1+\left.\lambda^{*}\right|_{\tau=t} & =0
\end{aligned}
$$

We obtain the lagrangian multiplier:

$\lambda^{*}=-1$

As a result, we obtain the following iteration formula:

$$
\begin{aligned}
\theta_{n+1}(z, t)= & \theta_{n}(z, t)- \\
& \int_{0}^{t}\left(\frac{\partial}{\partial \tau} \theta_{n}(z, \tau)+\theta_{n}(z, \tau) \frac{\partial}{\partial z} \theta_{n}(z, \tau)-\frac{\partial^{2}}{\partial z^{2}} \theta_{n}(z, \tau)\right) d \tau
\end{aligned}
$$

Now we start with an arbitrary initial approximation that satisfies the initial condition:

$\theta_{0}(z, t)=0.5-0.5 \tanh (0.25 z)$.

Using the above variational formula (49), we have:

$\theta_{1}(z, t)=\theta_{0}(z, t)-$

$$
{ }_{10} \quad \int_{0}^{t}\left(\frac{\partial}{\partial \tau} \theta_{0}(z, \tau)+\theta_{0}(z, \tau) \frac{\partial}{\partial z} \theta_{0}(z, \tau)-\frac{\partial^{2}}{\partial z^{2}} \theta_{0}(z, \tau)\right) \mathrm{d} \tau
$$

Substituting Eq. (50) in to Eq. (51) and after simplifications, we have:

$\theta_{1}(z, t)=\frac{0.0625\left(8 \cosh (0.25 z)^{2}-8 \sinh (0.25 z) \cosh (0.25 z)+t\right)}{\cosh (0.25 z)^{2}}$

\subsection{Case 2: if $n=2$}

To solve the Richards' equation by means of VIM, once again the following correction 15 functional may be constructed:

$\theta_{n+1}(z, t)=\theta_{n}(z, t)+\int_{0}^{t} \lambda^{*}\left(\frac{\partial}{\partial \tau} \theta_{n}(z, \tau)+\theta_{n}(z, \tau)^{2} \frac{\partial}{\partial z} \theta_{n}(z, \tau)-\frac{\partial^{2}}{\partial z^{2}} \theta_{n}(z, \tau)\right) \mathrm{d} \tau$ 
Its stationary conditions can be obtained as follows:

$$
\begin{aligned}
\left.\lambda^{\prime *}\right|_{\tau=t} & =0, \\
1+\left.\lambda^{*}\right|_{\tau=t} & =0,
\end{aligned}
$$

We obtain the lagrangian multiplier:

$5 \lambda^{*}=-1$

As a result, we obtain the following iteration formula:

$\theta_{n+1}(z, t)=\theta_{n}(z, t)-$

$$
\int_{0}^{t}\left(\frac{\partial}{\partial \tau} \theta_{n}(z, \tau)+\theta_{n}(z, \tau)^{2} \frac{\partial}{\partial z} \theta_{n}(z, \tau)-\frac{\partial^{2}}{\partial z^{2}} \theta_{n}(z, \tau)\right) \mathrm{d} \tau
$$

Now we start with an arbitrary initial approximation that satisfies the initial condition:

$10 \theta_{0}(z, t)=(0.5-0.5 \tanh (0.3333 z))^{0.5}$

Using the above variational formula (56), we have:

$$
\begin{aligned}
\theta_{1}(z, t)= & \theta_{0}(z, t)- \\
& \int_{0}^{t}\left(\frac{\partial}{\partial \tau} \theta_{0}(z, \tau)+\theta_{0}(z, \tau)^{2} \frac{\partial}{\partial z} \theta_{0}(z, \tau)-\frac{\partial^{2}}{\partial z^{2}} \theta_{0}(z, \tau)\right) \mathrm{d} \tau
\end{aligned}
$$

Substituting Eq. (57) in to Eq. (58) and after simplifications, we have:

$$
\begin{aligned}
& 15 \quad \theta_{1}(z, t)=-\frac{1}{(2-2 \tanh (0.3333 z))^{\frac{3}{2}}} \times
\end{aligned}
$$

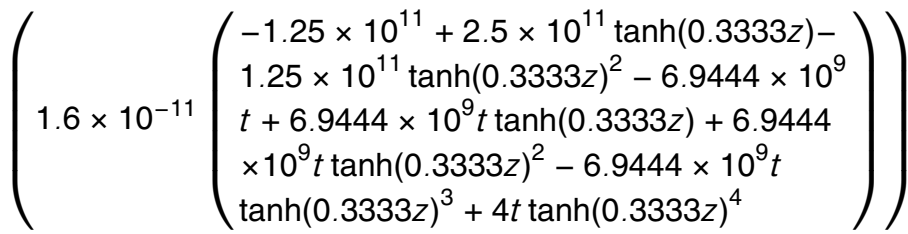

and so on. In the same way the rest of the components of the iteration formula can be obtained. Figures 1 and 2 show the results obtained from VIM and HPM along with the exact solution, revealing a high level of agreement between the three results shown. It should be noted that Richards' equation has significant physical meaning for

5 positive z-values. However, since there are some solutions given for infinite conditions in Richard's equation in the literature, i.e., for $-\infty<z<+\infty$ (especially for linear variations of $k$ ), solutions for negative values of $z$ have also been presented in the figures. It is evident from the curves plotted that the exact solution and the obtained solutions from HPM and VIM almost completely overlay each other and the level of agreement between the results is therefore excellent.

\section{Concluding remarks}

In this study, Analytical solution to Richards' equation was explored using Homotopy Perturbation Method (HPM) and Variational Iteration Method (VIM). Richards' equation is used for modeling infiltration in unsaturated soils. HPM and VIM have been success-

15 fully utilized for solving Richards' equation. Illustrative examples proved the high accuracy of the results obtained using HPM and VIM. Compared to the methods presented in the literature, the homotopy perturbation method is an asymptotic method in the sense that no convergence proof is needed for the solutions obtained, i.e. a converged solution is achieved within three iterations at the most, which means the method is quite robust in achieving the solution. Although the examples considered for Richards' equation herein involved one-dimensional cases, the success of the methods used are still appraisable, as it should be noted that the equation is still a nonlinear one even in its one-dimensional form. Following the success observed in one-dimensional cases, it is now hoped that more complex situations may also be addressed in the future. It can

25 be concluded that HPM and VIM may be effectively used for solving Richards' equation which covers some important class of problems in geotechnical and geo-environmental engineering. 


\section{References}

Abdou, M. A. and Soliman, A. A.: Modified extended Tanh-function method and its application on nonlinear physical equations, Phys. Lett. A, 353, 487-492, 2006. 6367

Arampatzis, G., Tzimopoulos, C., Sakellariou-Makrantonaki, M., and Yannopoulos, S.: Estima-

5 tion of unsaturated flow in layered soils with the finite control volume method, Irrig. Drain., 50, 349-358, 2001. 6360, 6361

Baca, R. G., Chung, J. N., and Mulla, D. J.: Mixed transform finite element method for solving the nonlinear equation for flow in variably saturated porous media, Int. J. Numer. Method. Fluid., 24, 441-455, 1997. 6361

10 Barari, A., Omidvar, M., Ghotbi, A. R., and Ganji, D. D.: Application of homotopy perturbation method and variational iteration method to nonlinear oscillator differential equations, Acta Appl. Math., 104, 161-171, 2008a. 6362, 6368

Barari, A., Ghotbi, A. R., Farrokhzad, F., and Ganji, D. D.: Variational iteration method and Homotopy-perturbation method for solving different types of wave equations, J. Appl. Sci., 8 , 120-126, 2008b. 6362, 6368

Barari, A., Omidvar, M., Ganji, D. D., and Tahmasebi Poor, A.: An Approximate solution for boundary value problems in structural engineering and fluid mechanics, J. Math. Problems Eng., Article ID 394103, 1-13, 2008c. 6362, 6368

Basha, H. A.: Multidimensional linearized nonsteady infiltration with prescribed boundary conditions at the soil surface, Water Resour. Res., 25(1), 75-93, 1999. 6361

Basha, H. A.: Burger's equation: a general nonlinear solution of infiltration and redistribution, Water Resour. Res., 38(11), 291-299, 2002. 6366

Bergamaschi, L. and Putti, M.: Mixed finite element and Newton-type linearizations for the solution of Richards' equation, Int. J. Numer. Method. Eng., 45, 1025-1046, 1999. 6361

25 Broadbridge, P. and Rogers, C.: Exact solution for vertical drainage and redistribution in soils, J. Eng. Math., 24(1), 25-43, 1990. 6366

Brooks, R. H. and Corey, A. T.: Hydraulic properties of porous media, Hydrology paper 3, Colorado State University, Fort Collins, 1964. 6361, 6365, 6366

Buckingham, E.: Studies on the movement of soil moisture, Bulletin 38, USDA Bureau of Soils,

$30 \quad$ Washington, DC, 1907. 6360, 6363

Bunsri, T., Sivakumar, M., and Hagare, D.: Numerical modeling of tracer transport in unsaturated porous media, J. Appl. Fluid Mech., 1(1), 62-70, 2008. 6361

6379

Chounet, L. M., Hilhorst, D., Jouron, C., Kelanemer, Y., and Nicolas, P.: Simulation of water flow and heat transfer in soils by means of a mixed finite element method, Adv. Water Resour., 22(5), 445-60, 1999. 6361

Clement, T. P., William, R. W., and Molz, F. J.: A physically based two-dimensional, finitedifference algorithm for modeling variably saturated flow, J. Hydrol., 161, 71-90, 1994. 6361

Corey, A. T.: Mechanics of immiscible fluids in porous Media. Water Resource Publications, Highlands Ranch, p. 252, 1994. 6365

Corey, A. T.: Mechanics of immiscible fluids in porous media. Water Resource Publications, Littleton, Colorado, 1986. 6366

10 Elwakil, S. A., El-Labany, S. K., Zahran, N. A., and Sabry, R.: Exact traveling wave solutions for diffusion-convection equation in two and three spatial dimensions, Computat. Phys. Commun., 158, 113-116, 2004. 6367

Farthing, M. W., Kees, C. E., Coffey, T. S., Kelley, C. T., and Miller, C. T.: Efficient steadystate solution techniques for variably saturated groundwater flow, Adv. Water Resour., 26(8), 833-849, 2003. 6361

Forsyth, P. A., Wu, Y.-S., and Pruess, K.: Robust numerical methods for saturated-unsaturated flow with dry initial conditions in heterogeneous media, Adv. Water Resour., 18, 25-38, 1995. 6361

Ghotbi, A. R., Avaei, A., Barari, A., and Mohammadzade, M. A.: Assessment of He's homotopy perturbation method in Burgers and coupled Burgers' equations, J. Appl. Sci., 8, 322-327, 2008a. 6362, 6368

Ghotbi, A. R., Barari, A., and Ganji, D. D.: Solving ratio-dependent predator-prey system with constant effort harvesting using homotopy perturbation method. J. Math. Prob. Eng., Article ID 945420, 1-8, 2008b. 6362, 6368

$25 \mathrm{He}, \mathrm{J}$. H.: A new approach to nonlinear partial differential equations, Commun. Nonlinear Sci., 2, 230-235, 1997. 6362, 6367, 6368

He, J. H.: Homotopy perturbation technique, Comput. Meth. Appl. Mech. Eng., 178, 257-262, 1999. 6362,6370

He, J. H.: Variational iteration method - a kind of non-linear analytical technique: some examples, Int. J. Nonlinear Mech., 34, 699-708, 1999. 6362, 6368

He, J. H.: A coupling method of a homotopy technique and a perturbation technique for nonlinear problems, Int. J. Nonlin. Mech., 35, 37-43, 2000. 6362, 6368

He, J. H.: Homotopy perturbation method: a new nonlinear analytical technique, Appl. Math. 
Comput., 135, 73-79, 2003. 6362

He, J. H.: Addendum: new interpretation of homotopy perturbation method, Int. J. Mod. Phys. B, 20(18), 2561-2568, 2006. 6362, 6368

$\mathrm{He}$, J. H.: Some asymptotic methods for strongly nonlinear equations, Int. J. Modern Phys. B,

$5 \quad 20,1141-1199,2006.6362,6368$

He, J. H. and Lee, E. W. M.: New analytical methods for cleaning up the solution of nonlinear equations. Comput. Math. Appl., in press, 2009. 6363

Hoffmann, M. R.: Macroscopic equations for flow in unsaturated porous media, Ph.D. dissertation, Washington University, 2003. 6360

10 Huang, K., Mohanty, B. P., and van Genuchten, M. T.: A new convergence criterion for the modified Picard iteration method to solve the variably saturated flow equation, J. Hydrol., 178, 69-91, 1996. 6361

Jones, J. E. and Woodward, C. S.: Newton-Krylov-multigrid solvers for large-scale, highly heterogeneous, variably saturated flow problems, Adv. Water Resour., 24(7), 763-74, 2001. 6361

Ju, S. H. and Kung, K. J. S.: Mass types, element orders and solution schemes for Richards' equation, in: Computers and Geosciences, 23(2), 175-187, 1997. 6360, 6361

Kavetski, D., Binning, P., and Sloan, S. W.: Adaptive backward Euler time stepping with truncation error control for numerical modeling of unsaturated fluid flow, Int. J. Numer. Method. Eng., 53, 1301-1322, 2001a. 6361

Kavetski, D., Binning, P., and Sloan, S. W.: Adaptive time stepping and error control in a mass conservative numerical solution of the mixed form of Richards' equation, Adv. Water Resour., 24, 595-605, 2001b. 6361

Kavetski, D., Binning, P., and Sloan, S. W.: Non-iterative time stepping schemes with adaptive

25 truncation error control for the solution of Richards' equation, Water Resour. Res., 38(10), 1211-1220, 2002. 6361

Kees, C. E. and Miller, C. T.: Higher order time integration methods for two-phase flow, Adv. Water Resour., 25(2), 159-77, 2002. 6361

Kevorkian, J. and Cole, J. D.: Multiple Scale and Singular Perturbation Methods, SpringerVerlag, New York, 1996. 6362

Miller, C. T., Abhishek, C., and Farthing, M. W.: A spatially and temporally adaptive solution of Richards' equation, Adv. Water Resour., 29(4), 525-545, 2005. 6361

Milly, P. C. D.: A mass conservation procedure for time-stepping in models of unsaturated flow,

6381

Adv. Water Resour., 8, 32-36, 1985. 6361

Momani, S. and Abuasad, S.: Application of He's variational iteration method to Helmholtz equation, Chaos Soliton Fract., 27, 1119-1123, 2006. 6362, 6368

Narasimhan, T. N.: Buckingham, 1907: an appreciation. Vadose Zone J., 4, 434-441, 2005. 6363

Nasseri, M., Shaghaghian, M. R., Daneshbod, Y., and Seyyedian, H.: An analytical Solution of Water Transport in Unsaturated Porous Media, J. Porous Med., 11(6), 591-601, 2008. 6367

Nayfeh, A. H.: Perturbation Methods, John Wiley \& Sons, New York, 1973. 6362

Nayfeh, A. H. and Mook, D. T.: Nonlinear Oscillations, John Wiley \& Sons, New York, 1979. 6362

Philip, J. R.: Fifty years progress in soil physics, Geoderma, 12, 265-280, 1974. 6364

Richards, L. A.: Capillary conduction of liquids through porous mediums, Physics, 1, 318-333, 1931. 6360, 6363

Rolston, D. E.: Historical development of soil-water physics and solute transport in porous media, Water Sci. Technol., 7, 1, 59-66, 2007.

Ross, P. J.: Modeling soil water and solute transport - fast, simplified numerical solutions, Agr. J., 95, 1352-1361, 2003. 6361

Soliman, A. A.: The modified extended tanh-function method for solving Burgers-type equations, Physica A, 361, 394-404, 2006. 6367

20 Sweilam, N. H. and Khader, M. M.: Variational iteration method for one dimensional nonlinear thermo-elasticity, Chaos Soliton Fract., 32, 145-149, 2007. 6362, 6368

Tabuada, M. A., Rego, Z. J. C., Vachaud, G., and Pereira, L. S.: Two-dimensional irrigation under furrow irrigation: modeling, its validation and applications, Agr. Water Manage., 27(2), 105-123, 1995. 6361

25 Van Genuchten, M. T.: A closed-form equation for predicting the hydraulic conductivity of unsaturated soils, J. Soc. Soil Sci. Am., 44, 892-898, 1980. 6365

Varado, N., Braud, I., Ross, P. J., and Haverkamp, R.: Assessment of an efficient numerical solution of the 1D Richards equation on bare soil, J. Hydrol., 323(1-4), 244-257, 2006. 6361

Wazwaz, A. M.: Traveling wave solutions for generalized forms of Burgers, Burgers-KDV and

30 Burgers-Huxley equations, Appl. Math. Comput., 169, 639-656, 2005. 6367

Witelski, T. P.: Perturbation analysis for wetting fronts in Richards' equation, Transport Porous Med., 27(2), 121-134, 1997. 6362, 6366, 6367, 6368

Witelski, T. P.: Motion of wetting fronts moving into partially pre-wet soil, Adv. Water Resour., 
28, 1131-1141, 2005. 6366

Whitman, G. B.: Linear and nonlinear waves. New York, John Wiley and Sons, 1974. 6366, 6367

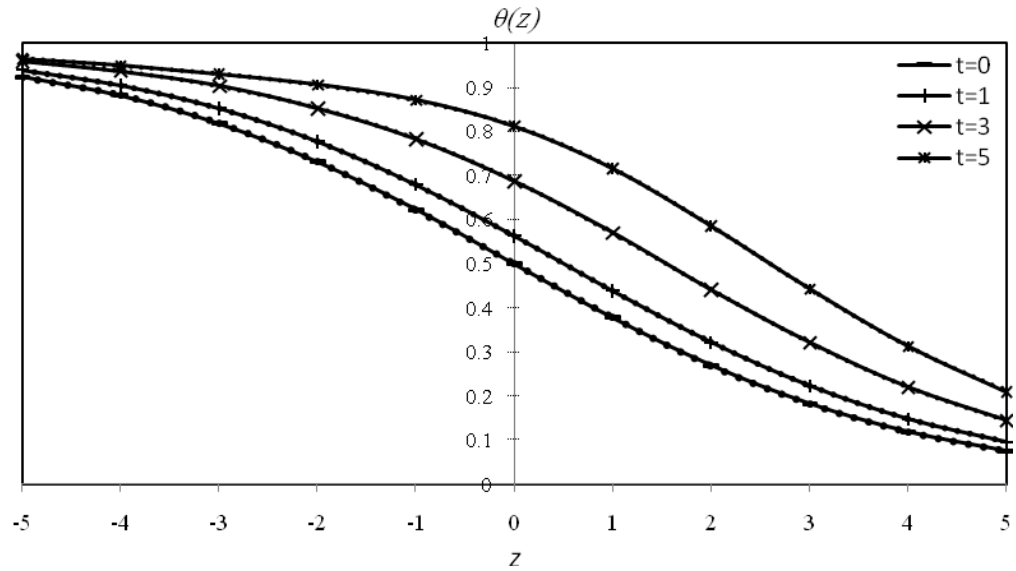

Fig. 1. Plot of $\theta(z)$ for different values of time $(t=0,1,3,5)$ considering $n=1$ - solid lines represent results from VIM and HPM, while dashed lines depict the exact solution. 


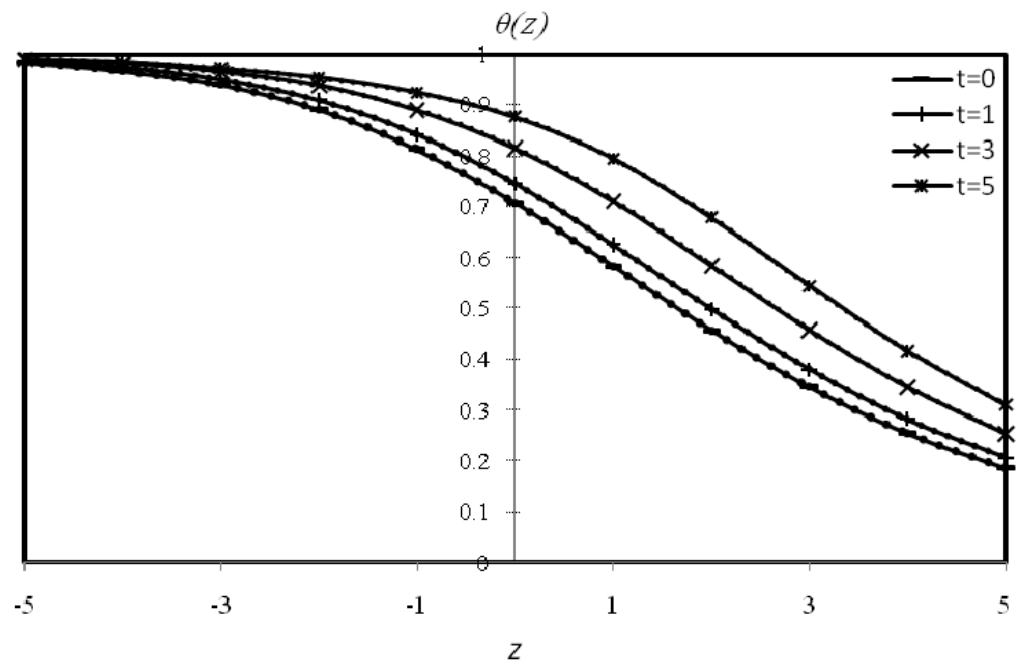

Fig. 2. Plot of $\theta(z)$ for different values of time $(t=0,1,3,5)$ considering $n=2-$ solid lines represent results from VIM and HPM, while dashed lines depict the exact solution. 